\title{
"Soft" Anharmonic Vortex Glass in Ferromagnetic Superconductors
}

\author{
Leo Radzihovsky, ${ }^{1}$ A. M. Ettouhami, ${ }^{1}$ Karl Saunders, ${ }^{2}$ and John Toner $^{2}$ \\ ${ }^{1}$ Department of Physics, University of Colorado, Boulder, Colorado 80309 \\ ${ }^{2}$ Department of Physics, Materials Science Institute, and Institute of Theoretical Science, \\ University of Oregon, Eugene, Oregon 97403
}

(Received 9 March 2001; published 21 June 2001)

\begin{abstract}
Ferromagnetic order in superconductors can induce a spontaneous vortex (SV) state. For external field $\mathbf{H}=0$, rotational symmetry guarantees a vanishing tilt modulus of the SV solid, leading to drastically different behavior than that of a conventional, external-field-induced vortex solid. We show that quenched disorder and anharmoinc effects lead to elastic moduli that are wave-vector dependent out to arbitrarily long length scales, and non-Hookean elasticity. The latter implies that for weak external fields $H$, the magnetic induction scales universally like $B(H) \sim B(0)+c H^{\alpha}$, with $\alpha \approx 0.72$. For weak disorder, we predict the SV solid is a topologically ordered glass, in the "columnar elastic glass" universality class.
\end{abstract}

DOI: $10.1103 /$ PhysRevLett.87.027001

PACS numbers: $74.25 . \mathrm{Ha}, 74.40 .+\mathrm{k}$

Rare-earth borocarbide materials exhibit a rich phase diagram that includes superconductivity, antiferromagnetism, ferromagnetism, and spiral magnetic order [1-3]. In particular, there is now ample experimental evidence that, at low temperatures, superconductivity and ferromagnetism competitively coexist in $\mathrm{ErNi}_{2} \mathrm{~B}_{2} \mathrm{C}$ compounds. Other possible examples of such ferromagnetic superconductors (FS) are the recently discovered high temperature superconductor $\mathrm{Sr}_{2} \mathrm{YRu}_{1-x} \mathrm{Cu}_{x} \mathrm{O}_{6}$ and the putative $p$-wave triplet strontium ruthenate superconductor, $\mathrm{Sr}_{2} \mathrm{RuO}_{4}$, which spontaneously breaks time reversal symmetry. For sufficiently strong ferromagnetism, such FS's have been predicted [3] to exhibit a spontaneous vortex (SV) state driven by the spontaneous magnetization, rather than by an external magnetic field $\mathbf{H}$. The novel phenomenology of the associated SV solid is the subject of this Letter.

Here we will show that for $\mathbf{H}=0$, the elastic properties of the resulting SV solid differ dramatically and qualitatively from those of a conventional Abrikosov lattice. The key underlying difference is the vanishing of the tilt modulus, which is guaranteed by the underlying rotational invariance (but see below). Although this invariance is broken by the magnetization, $\mathbf{M}$, the tilt modulus remains zero because this breaking is spontaneous. This contrasts strongly with a conventional vortex solid, where the rotational symmetry is explicitly broken by the applied field H. All of our conclusions, e.g., the unusual $B(H)$ relation illustrated in Fig. 1, are a direct consequence of this important observation.

In particular, we find that this "softness" (i.e., vanishing tilt modulus) of the SV lattice drastically enhances the effects of quenched disorder. As in conventional vortex lattices [4], any amount of disorder $\Delta_{V}$, however weak, is sufficient to destroy translational order in SV lattices. Here the finite ordered domains are divergently anisotropic, with dimensions $\xi_{\perp}^{L} \propto 1 / \Delta_{V}^{2 / 3}$ and $\xi_{z}^{L} \propto 1 / \Delta_{V}^{1 / 3}$. These lengths are measurable in scattering and transport measurements.
Unlike conventional lattices, however, in SV lattices the disorder also qualitatively alters the elastic behavior at long distances, leading to "anomalous elasticity": a universal scaling of elastic moduli with wave vector $\mathbf{k}$ out to arbitrarily long length scales, with some elastic moduli vanishing, and others diverging, as wave vector $\mathbf{k} \rightarrow 0$ [5]. This behavior is characteristic of a new kind of topologically ordered "columnar elastic glass"(CEG) phase of vortices, which is stable, for weak disorder, against proliferation of dislocations.

The best way to experimentally probe a spontaneously broken symmetry is to break it directly with an external field. We predict that as a consequence of the anomalous elasticity, the increase in the magnetic induction, $\delta B(H) \equiv$ $B(H)-B(0)$ over the spontaneous induction $B(0)$, due to a weak applied field $\mathbf{H}$ along $\mathbf{M}$ obeys a universal

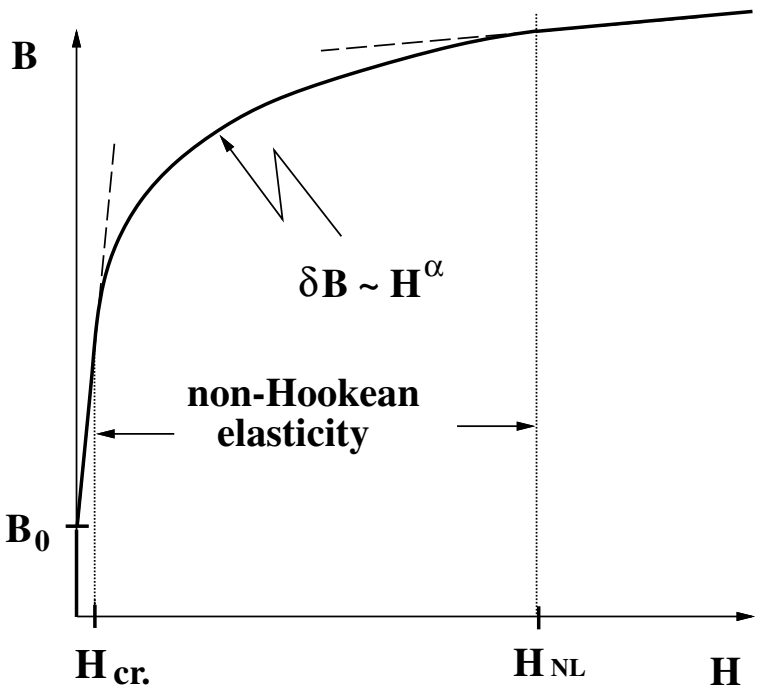

FIG. 1. The nonlinear and universal power-law $B(H)$ scaling, which at weakest fields $H<H_{\mathrm{cr}}$ and strongest fields $H>H_{\mathrm{NL}}$ is cut off by the crystal symmetry breaking anisotropy and $\xi_{\mathrm{NL}}$, respectively. 
"non-Hookean" scaling law:

$$
\delta B(H) \propto|H|^{\alpha},
$$

with the universal exponent $\alpha=0.72 \pm 0.04$, a prediction that should be experimentally testable.
Whatever the microscopic origin of the novel FS state, general symmetry principles dictate that the long length scale phenomenology is described by an effective LandauGinzburg free energy functional

$$
\begin{aligned}
F_{\mathrm{GL}}=\int d^{3} r[ & \frac{\hbar^{2}}{2 m}\left|\left(\nabla-i \frac{2 \pi}{\phi_{0}} \mathbf{A}\right) \psi\right|^{2}+\frac{a}{2}|\psi|^{2}+\frac{b}{4}|\psi|^{4}+\frac{K}{2}|\nabla \mathbf{M}|^{2}+\frac{t}{2}|\mathbf{M}|^{2}+\frac{u}{4}|\mathbf{M}|^{4} \\
& \left.+\frac{1}{8 \pi}|\mathbf{B}-4 \pi \mathbf{M}|^{2}-\frac{1}{4 \pi} \mathbf{B} \cdot \mathbf{H}\right],
\end{aligned}
$$

where $\psi$ is the superconducting order parameter and $\mathbf{A}$ is the electromagnetic vector potential with $\mathbf{B}=\boldsymbol{\nabla} \times \mathbf{A}$. The constants $m, a, b, K, t$, and $u$ are experimentally measurable phenomenological parameters, with $a$ and $t$ changing their signs at the superconducting and ferromagnetic decoupled transition temperatures, respectively, and $\phi_{0}=h c / 2 e$ the elementary magnetic flux quantum.

The above model displays rich phenomenology [3]. It has been shown in detail by Greenside et al. [3] that for a range of physically realistic parameters [e.g., $\lambda_{L} / \xi$, $\lambda_{L} / \sqrt{K /|t|} \approx O(10)$ [3], with the large Abrikosov ratio required for a robust mixed state, and large exchange $K$ necessary to suppress the competing spiral phase, relative to the SV state], systems described by $F_{\mathrm{GL}}$, Eq. (2), exhibit a thermodynamically stable phase consisting of a spontaneous (i.e., $\mathbf{H}=0$ ) vortex state, in which superconducting vortices are generated by the spontaneous magnetization of the ferromagnetic component.

Deep within this state, both $\psi(\mathbf{r})$ and $\mathbf{M}(\mathbf{r})$ are large, and therefore the London approximation applies. We take $\mathbf{M}(\mathbf{r})=M_{0} \hat{\mathbf{n}}(\mathbf{r})$ to be of approximately uniform magnitude $M_{0}$ and fluctuating direction $\hat{\mathbf{n}}(\mathbf{r})$. We also take $|\psi(\mathbf{r})|$ to be a constant $\psi_{0} \neq 0$, everywhere except at the locations of the vortices (where $\psi=0$ ) and allow its phase $\theta(\mathbf{r})$ to vary subject to the circulation condition that it increases by an amount $2 \pi$ along a closed path enclosing a vortex [6]. The magnetic induction $\mathbf{B}(\mathbf{r})$ is confined to thin flux filaments defined by the zeros of $\psi$. Using the circulation condition and the minimizing above free energy $F_{\mathrm{GL}}$ with respect to $\psi_{0}, \theta$, and $\mathbf{A}$, we eliminate $\boldsymbol{\nabla} \theta$ and $\mathbf{B}$ in favor of vortex line conformational degrees of freedom and obtain

$$
\begin{aligned}
F_{L}= & \frac{\phi_{0}^{2}}{8 \pi} \int d^{3} r d^{3} r^{\prime} \mathbf{t}(\mathbf{r}) \cdot \mathbf{t}\left(\mathbf{r}^{\prime}\right) V\left(\left|\mathbf{r}-\mathbf{r}^{\prime}\right|\right) \\
& +\int d^{3} r\left[\frac{1}{2} K M_{0}^{2}|\nabla \hat{\mathbf{n}}|^{2}-\phi_{0} M_{0} \mathbf{t}(\mathbf{r}) \cdot \hat{\mathbf{n}}\right],
\end{aligned}
$$

where we have dropped unimportant constant pieces. $\mathbf{t}(\mathbf{r})=\sum_{n} \int d s \frac{d \mathbf{r}_{n}}{d s} \delta^{3}\left[\mathbf{r}-\mathbf{r}_{n}(s)\right]$ is the directed vortex line density, with magnitude given by the local flux density (in units of $\phi_{0}$ ) and with direction tangent to the local vorticity; $\mathbf{r}_{n}(s)$ is the locus of the $n$th vortex line, parametrized by an arc length $s$. The vortex interaction $V(r)$ is approximately given by the standard expression $V(r)=$ $e^{-r / \lambda_{L}} /\left(4 \pi \lambda_{L}^{2} r\right)$, with $\lambda_{L}^{2}=m \phi_{0}^{2} /\left(16 \pi^{3} \hbar^{2}\left|\psi_{0}\right|^{2}\right)$ [3], cut off at scales smaller than the coherence length $\xi$.

At low temperature $T$ and in the clean limit the vortex lines freeze into a lattice (as has been observed [1]), directed on average along a spontaneously chosen magnetization direction $\hat{\mathbf{n}}_{0}$, which we choose to be the $\hat{\mathbf{z}}$ axis, and with the lattice constant determined by the average magnetization $M_{0}$. Vortex lattice fluctuations are described by the 2D phonon displacement vector field $\mathbf{u}\left(z, \mathbf{R}_{n}\right)$, defined by $\mathbf{r}_{n}(z)=\left(X_{n}+u_{x}\left(z, \mathbf{R}_{n}\right), Y_{n}+u_{y}\left(z, \mathbf{R}_{n}\right), z\right)$, where $\mathbf{R}_{n}=\left(X_{n}, Y_{n}\right)$ spans the vortex lattice, and we chose $s=z$, and by a small 2D vector $\delta \mathbf{n} \perp \hat{\mathbf{z}}$ defined by $\hat{\mathbf{n}}=(1-\delta \mathbf{n} \cdot \delta \mathbf{n})^{1 / 2} \hat{\mathbf{z}}+\delta \mathbf{n}$.

Integrating out $\delta \mathbf{n}$ and going to the continuum, we obtain $[7,8]$ the effective elastic Hamiltonian:

$$
H_{\mathrm{el}}=\frac{1}{2} \int d^{3} r\left[\kappa\left|\partial_{z}^{2} \mathbf{u}\right|^{2}+2 \mu u_{i j}^{2}+\lambda u_{i i}^{2}\right],
$$

where $\kappa$ is the vortex line curvature modulus and $\mu$ and $\lambda$ are the Lamé coefficients. $\mu, \lambda$, and $\kappa$ are all expressible in terms of the parameters of the original model Eq. (2), and $u_{i j}=\frac{1}{2}\left(\partial_{i} u_{j}+\partial_{j} u_{i}-\partial_{z} u_{i} \partial_{z} u_{j}\right)$ is the rotationally invariant nonlinear strain tensor, with $i, j \in x, y$. This form of $H_{\mathrm{el}}$ is strictly dictated by general symmetry considerations, specifically: the underlying rotational symmetry of the SV solid about any arbitrary axis guarantees that only the fully rotationally invariant combination $u_{i j}$, given above, appears and that the vortex line tilt modulus $\left(c_{44}\right)$ vanishes identically (but see below) [8].

Although the softness of the SV lattice suggests, via the Lindemann criterion, a lower melting temperature, our long wavelength description cannot directly predict the melting temperature, because thermal fluctuations are dominated by short distance modes (near the Brillouin zone boundary). Instead, here we focus on the much more interesting, robust, and universal effects of quenched disorder, all of which (at long wavelengths) can be incorporated into our model by adding to $H_{\mathrm{el}}$

$$
H_{\mathrm{dis}}=\int d^{3} r\left[\mathcal{R} e \sum_{\mathbf{G}} V_{\mathbf{G}}(\mathbf{r}) e^{i \mathbf{G} \cdot \mathbf{u}(\mathbf{r})}+\mathbf{h}(\mathbf{r}) \cdot \partial_{z} \mathbf{u}\right] \text {. }
$$

The first term accounts for the random positional pinning (at reciprocal lattice vectors $\mathbf{G}$ ) of the vortex lattice, with $V_{\mathbf{G}}(\mathbf{r})$ a complex random pinning potential, whose statistics can be accurately represented by zero-mean, short-ranged, Gaussian correlations: $\overline{V_{\mathbf{G}}(\mathbf{r}) V_{\mathbf{G}^{\prime}}^{*}\left(\mathbf{r}^{\prime}\right)}=\Delta_{V} \delta_{\mathbf{G}_{\mathbf{G}^{\prime}}}^{\perp} \delta^{d}\left(\mathbf{r}-\mathbf{r}^{\prime}\right)$ [9]. The second term reflects the presence of an additional orientational, tilt disorder. The tilt field $\mathbf{h}(\mathbf{r})$ is a random vector 
with short-ranged isotropic spatial correlations, whose statistics can also be taken zero-mean Gaussian, with $\overline{h_{i}(\mathbf{r}) h_{j}\left(\mathbf{r}^{\prime}\right)}=\Delta \delta_{i j} \delta^{d}\left(\mathbf{r}-\mathbf{r}^{\prime}\right)$ [9]. In the case of magnetic impurities, it is the random local fields, acting on $\mathbf{M}$, i.e., $\mathbf{h}(\mathbf{r}) \cdot \hat{\mathbf{n}}$, which via the Higg's mechanism, $\delta \mathbf{n} \approx \partial_{z} \mathbf{u}$, lead to the tilt disorder piece of $H_{\mathrm{dis}}$. In the case of nonmagnetic impurities, it is the short-scale anisotropy in the spatial distribution of vortex line pinning centers, together with lack of rotational symmetry in the SV state, that leads to the tilt disorder in Eq. (5), as can be easily illustrated by short-scale modes thinning procedure [8]. (As in conventional vortex lattices, in $d>2$, the pinning of the long-scale vortex density distortions is irrelevant at long scales.)

Adapting Larkin's [4] calculation to the "soft" elasticity of the SV lattice we find that in $d$ dimensions and at short length scales, mean-squared lattice distortions [in domains of size $\left.L_{\perp}\left(\ll L_{z}\right)\right]$ grow as $u_{\mathrm{rms}}^{2} \sim L_{\perp}^{9 / 2-d}$. Hence, translational order is destroyed for $d=3<d_{c}^{\Delta_{V}}=9 / 2$, implying (in the weak disorder limit) divergently anisotropic Larkin domains spanned by 3D Larkin lengths $L_{\perp}^{L} \approx\left[a^{2}\left(\kappa B_{s}^{7}\right)^{1 / 4} / \Delta_{V}\right]^{2 / 3}$ and $L_{z}^{L} \approx\left(a^{2} \kappa B_{s} / \Delta_{V}\right)^{1 / 3}$, with $a$ the vortex spacing, $s \in L, T$, and $B_{L}=2 \mu+\lambda$, $B_{T}=\mu$. Beyond these Larkin length scales, the Larkin approximation breaks down and the full nonlinear nature of the random-field pinning potential must be taken into account, leading to much more weakly (specifically, logarithmically) divergent lattice fluctuations as in conventional vortex lattices [10]. This weak divergence is completely overwhelmed for $d<d_{u c}=7 / 2$ by the far stronger fluctuations induced by the tilt disorder, which diverge algebraically: $u_{\mathrm{rms}}^{2} \sim L_{\perp}^{7 / 2-d}$ (ignoring nonlinear elastic effects) out to arbitrarily long length scales $[8,9,11,12]$.

Ignoring nonlinear elastic terms, we obtain the disorderaveraged phonon correlation function $\overline{C_{s}(\mathbf{r})} \approx$ $\operatorname{Max}\left[\Delta /\left(\kappa^{3} B_{s}^{5}\right)^{1 / 4} r_{\perp}^{7 / 2-d}, \Delta /\left(\kappa^{5-d} B_{s}^{d-1}\right)^{1 / 2} z^{7-2 d}\right] \times O(1)$. The spatial (Larkin-like) correlation lengths, measurable in neutron scattering experiments, are defined by $C_{s}\left(\operatorname{Min}\left[\xi_{\perp}, \xi_{z}\right]\right)=a^{2}$ and are given by $\xi_{\perp} \approx$ $\left[a^{2}\left(\kappa^{3} B_{s}^{5}\right)^{1 / 4} / \Delta\right]^{1 /(7 / 2-d)}, \quad \xi_{z} \approx \xi_{\perp}^{1 / 2}\left(\kappa / B_{s}\right)^{1 / 4}$. Hence, in $3 \mathrm{D}$, in the presence of arbitrarily weak disorder $\Delta$, on length scales longer than $\xi_{\perp, z}$ the translational order of SV solid decays rapidly; that is, it is short ranged. This, however, does not imply that the system is equivalent to the SV liquid state, or to a fully disordered vortex glass as it would be if dislocation loops unbound. Using duality methods, introduced recently by two of the authors [9], we have shown that a translationally disordered, but topologically ordered CEG phase is, in fact, possible in 3D. This is analogous to our recent results in the context of randomly pinned smectic liquid crystals $[9,11]$ and columnar phase of discotic liquid crystals [12] confined in aerogel, as well as the Bragg glass conjecture for conventional vortex lattices [13] and the random field $X Y$ model [14].
In this topologically ordered phase the anharmonic elastic terms in the Hamiltonian lead to drastically modified elastic scaling on length scales longer than the nonlinear crossover length scales $\xi_{\mathrm{NL}}^{z}=8 \pi^{2} \kappa^{2} / \Delta$ and $\xi_{\mathrm{NL}}^{\perp}=\left(\xi_{\mathrm{NL}}^{z}\right)^{2}\left(B_{s} / \kappa\right)^{1 / 2}$. In renormalization group (RG) language, on scales longer than $\xi_{\mathrm{NL}}^{z, \perp}$, our system flows away from a zero-disorder Gaussian fixed point and approaches a nontrivial $T=0$ anharmonic, disordered fixed point. This new fixed point can be studied using standard RG analysis [9], and we predict that at scales longer than $\xi_{\mathrm{NL}}^{z, \perp}$, out to an arbitrary length scale (but see below), the elastic moduli $\kappa, \mu, \lambda$, and the disorder variance $\Delta$ become singular functions of wave vector $\mathbf{k}$ [5]:

$$
\begin{aligned}
& \kappa(\mathbf{k})=\kappa_{0}\left(k_{z} \xi_{\mathrm{NL}}^{z}\right)^{-\eta_{\kappa}} f_{\kappa}\left(k_{\perp} \xi_{\mathrm{NL}}^{\perp} /\left(k_{z} \xi_{\mathrm{NL}}^{z}\right)^{\zeta}\right) \\
& \mu(\mathbf{k})=\mu_{0}\left(k_{z} \xi_{\mathrm{NL}}^{z}\right)^{\eta_{\mu}} f_{\mu}\left(k_{\perp} \xi_{\mathrm{NL}}^{\perp} /\left(k_{z} \xi_{\mathrm{NL}}^{z}\right)^{\zeta}\right) \\
& \lambda(\mathbf{k})=g_{*} \mu_{0}\left(k_{z} \xi_{\mathrm{NL}}^{z}\right)^{\eta_{\mu}} f_{\mu}\left(k_{\perp} \xi_{\mathrm{NL}}^{\perp} /\left(k_{z} \xi_{\mathrm{NL}}^{z}\right)^{\zeta}\right) \\
& \Delta(\mathbf{k})=\Delta_{0}\left(k_{z} \xi_{\mathrm{NL}}^{z}\right)^{1-2 \eta_{\kappa}} f_{\Delta}\left(k_{\perp} \xi_{\mathrm{NL}}^{\perp} /\left(k_{z} \xi_{\mathrm{NL}}^{z}\right)^{\zeta}\right)
\end{aligned}
$$

where $f_{\kappa / \mu / \Delta}(x)$ is independent of $x$ if $x \ll 1$ and goes like $x$ to the power $-\eta_{\kappa} / \zeta, \eta_{\mu} / \zeta$, and $\left(1-2 \eta_{\kappa}\right) / \zeta$, respectively, when $x \gg 1$, so that, e.g., $\kappa(\mathbf{k}) \propto k_{\perp}^{-} \eta_{\kappa} / \zeta$ for $k_{\perp} \xi_{\mathrm{NL}}^{\perp} \gg\left(k_{z} \xi_{\mathrm{NL}}^{z}\right)^{\zeta}$. $\kappa_{0}, \mu_{0}$, and $\Delta_{0}$ are the bare values of the elastic constants and the anomalous exponents are universal, and to leading order in $\epsilon=\frac{7}{2}-d$ are given by $\eta_{\kappa}=1.478 \epsilon=0.74$ and $\eta_{\mu}=0.6919 \epsilon=$ 0.346 , where the second equalities are their values in 3D. The constant $g_{*}$ is also universal, and to leading order in $\epsilon$ $g_{*}=-0.03272+O(\epsilon)$. The anisotropy exponent $\zeta=$ $2-\left(\eta_{\kappa}+\eta_{\mu}\right) / 2$, and therefore the two independent exponents $\eta_{\kappa}$ and $\eta_{\mu}$ completely characterize the anomalous elasticity, with $\kappa(\mathbf{k})$ and $\Delta(\mathbf{k})$ diverging with linearly related exponents, and $\mu(\mathbf{k})$ and $\lambda(\mathbf{k})$ both vanishing in exactly the same way as $\mathbf{k} \rightarrow \infty$. The negative universal amplitude ratio $g_{*}$ implies that the SV glass exhibits a negative universal Poisson ratio $\sigma_{p}=-0.0338+O(\epsilon)$.

For length scales longer than $\xi_{\mathrm{NL}}$ this strong, power-law anomalous elasticity will alter the behavior of all physical observables of the SV solid, such as, e.g., the width of the structure function peak $S(\mathbf{k})$ measured in neutron scattering and the behavior in its tails. It also leads to a non-Hookean, i.e., nonlinear, stress-strain relation even for arbitrarily small applied stress $\sigma$.

To see this, consider a purely compressive stress, $\sigma_{i j}=$ $\sigma \delta_{i j}$, which adds a term $\sigma \boldsymbol{\nabla} \cdot \mathbf{u}=\sigma u_{i i}+\frac{1}{2}\left|\partial_{z} \mathbf{u}\right|^{2}$ to the Hamiltonian. In Fourier space, the second, symmetry breaking, term becomes $\sigma k_{z}^{2}|\mathbf{u}(\mathbf{k})|^{2}$ and begins to dominate over the $\kappa(\mathbf{k}) k_{z}^{4}|\mathbf{u}(\mathbf{k})|^{2}$ term once $\sigma k_{z}^{2} \geq \kappa(\mathbf{k}) k_{z}^{4}$. This clearly happens for $k_{z}$ 's less than a critical $k_{c}$ given by $\sigma k_{c}^{2}=\kappa\left(k_{z}=k_{c}, \mathbf{k}_{\perp}=0\right) k_{c}^{4}$. Using Eq. (6a) for $\kappa(\mathbf{k})$ in this expression and solving for $k_{c}$, we find $k_{c}(\sigma)=\left(\sigma / \kappa_{0}\right)^{1 /\left(2-\eta_{\kappa}\right)}\left(\xi_{\mathrm{NL}}^{z}\right)^{\eta_{\kappa} /\left(2-\eta_{\kappa}\right)}$.

Now, for sufficiently weak stress $\sigma, 1 / \xi_{\mathrm{NL}}^{z} \gg k_{c}(\sigma)$, the stress-induced rotational symmetry breaking term is subdominant to the vortex curvature energy $\kappa(\mathbf{k}) k_{z}^{4}|\mathbf{u}(\mathbf{k})|^{2}$ 
for wave vectors $k_{z} \gg k_{c}(\sigma)$. Consequently, on intermediate length scales, $k_{c}(\sigma)<k_{z}<k_{\mathrm{NL}}^{z}$, the SV glass exhibits anomalous elasticity summarized by Eqs. (6). However, on longer length scales, $k_{z} \ll k_{c}(\sigma)$, the applied stress energy dominates, suppressing fluctuations and cutting off the anomalous elasticity at $k_{c}(\sigma)$. Therefore, the elastic moduli saturate, for $k_{z} \ll k_{c}(\sigma)$, at their values at $k_{z}=k_{c}(\sigma), k_{\perp}=0$ given by Eq. (6), i.e., $\mu(\mathbf{k} \rightarrow 0) \rightarrow \mu\left(k_{z}=k_{c}, \mathbf{k}_{\perp}=0\right) \propto k_{c}^{\eta_{\mu}}$ and $\lambda(\mathbf{k} \rightarrow 0) \rightarrow$ $\lambda\left(k_{z}=k_{c}, \mathbf{k}_{\perp}=0\right) \propto k_{c}^{\eta_{\mu}}$. This implies that the Young's modulus $Y(\sigma)=4 \mu(\mu+\lambda) /(2 \mu+\lambda)=4 \mu_{0}\left(1+g_{*}\right) /$ $\left(2+g_{*}\right)\left(k_{c} \xi_{\mathrm{NL}}^{z}\right)^{\eta_{\mu}} \approx 2 \mu_{0}\left(k_{c} \xi_{\mathrm{NL}}^{z}\right)^{\eta_{\mu}}=2 \mu_{0}\left(\sigma / \sigma_{\mathrm{NL}}\right)^{\beta}$, with $\sigma \ll \sigma_{\mathrm{NL}}, \beta=\eta_{\mu} /\left(2-\eta_{\kappa}\right)$, and $\sigma_{\mathrm{NL}}=\kappa_{0} \xi_{\mathrm{NL}}^{-2}$. Hence, we find for stress $\sigma \leq \sigma_{\mathrm{NL}}$, a nonlinear (nonHookean) strain-stress relation: $\epsilon_{c}(\sigma) \equiv \boldsymbol{\nabla} \cdot \mathbf{u}=\sigma / Y(\sigma) \propto$ $\sigma^{\alpha}$, with $\alpha=1-\beta=1-\eta_{\mu} /\left(2-\eta_{\kappa}\right) \approx 0.72$.

Now, it is easy to see that $\mathbf{H}=H \hat{z}$, applied along the direction of the SV lattice, acts as a compressive stress, $\sigma=$ $H B(0)$, and the induced $\delta B(H)=B(H)-B(0)$ plays the role of the compressive strain, $\epsilon_{c}=\delta B / B(0)$. Hence $H$ allows us to directly probe the anomalous elasticity, with the non-Hookean elasticity leading to the nonlinear and universal $B(H)$ relation, for $H<H_{\mathrm{NL}}=\sigma_{\mathrm{NL}} / B(0)$, given in Eq. (1) and Fig. 1.

Of course, as with all crystalline ferromagnets, crystalline symmetry breaking fields will explicitly break rotational invariance, even in the absence of H. They will lead to a nonzero tilt modulus, i.e., a term ${ }_{2}^{1} V_{\mathrm{cr}}\left|\partial_{z} \mathbf{u}\right|^{2}$ in the Hamiltonian, which will cut off the anomalous elasticity on length scales longer than $\xi_{\text {cr }}^{z}=\left(\begin{array}{c}\kappa_{0} \\ V_{\text {cr }}\end{array}\right)^{1 /\left(2-\eta_{k}\right)}\left(\xi_{\mathrm{NL}}^{z}\right)^{-\eta_{\kappa} /\left(2-\eta_{\kappa}\right)}$. For weak $V_{\text {cr }}$, our predictions will apply over a wide range of length scales $L_{z, \perp}$ satisfying $\xi_{\mathrm{cr}}^{z, \perp} \gg L_{z, \perp} \gg \xi_{\mathrm{NL}}^{z, \perp}$. On longer length scales the SV solid elasticity will cross over to the conventional "tension" elasticity of vortex lattices [6], exhibiting a linear relation $\delta B \propto H$, for $H<H_{\mathrm{cr}}=V_{\mathrm{cr}} / B(0)$.

The crystal anisotropy also leads to metastability for $|H|<H_{\mathrm{cr}}$, thereby allowing experimental studies of $\mathbf{H}$ applied in the direction opposite to that of $\mathbf{M}$. In the limit $|H| \rightarrow H_{\mathrm{cr}}$, the vortex solid becomes soft again, with the crystal-field generated tilt modulus vanishing as $H_{\mathrm{cr}}-|H|$, and the system exhibiting anomalous elasticity out to arbitrary length scale at this finely field-tuned point. However, because the rotational symmetry is nevertheless broken [with restoration only at the quadratic $\left(\partial_{z} \mathbf{u}\right)^{2}$ level], we expect that the universality class and therefore the anomalous exponents will be distinct from that of the CEG studied here [8].

As argued in the case of vanishing crystal anisotropy, here, too, the anomalous elasticity leads to a nontrivial field dependence of the Young's modulus. We find $Y(H) \propto$ $\left(H_{\mathrm{cr}}-|H|\right)^{\beta^{\prime}}$, with $\beta^{\prime}>0$ a universal exponent analogous to, but distinct from, the $\beta$ of the CEG. However, in contrast to the rotationally invariant case, here the flux density $\delta B(H)=H / Y(H)=H\left(H_{\mathrm{cr}}-|H|\right)^{-\beta^{\prime}}$ diverges as $|H|$ approaches $H_{\text {cr }}$ from below. This signals a break- down of the analysis, which assumes $\delta B(H) \ll B(0)$, and suggests that the metastability limit occurs at fields $H_{\mathrm{ms}}$ determined by $\delta B\left(H_{\mathrm{ms}}\right) \approx B(0)$.

Finally, we note that $\mathrm{ErNi}_{2} \mathrm{~B}_{2} \mathrm{C}$ has a strong uniaxial anisotropy, with $\mathbf{M}$ preferentially lying in a nearly isotropic $x z$ plane. The appropriate model in this case resembles that given by Eq. (4), except that in the strain tensor $u_{i j}$, $u_{i} \rightarrow u_{x}$, and $u_{y}$ has a conventional quadratic, tension elasticity. A similar model has recently been considered by us in the context of the columnar phase of discotics confined in an anisotropic random medium, such as, e.g., strained aerogel [12]. The resulting " $m=1$ elastic glass" phase is also topologically stable with anomalous elasticity, and has additional interesting features, most notably that it exhibits short-ranged translational order in one direction $(x)$ and quasi-long-ranged translational order in the other $(y)$, a prediction that should be testable via neutron scattering and decoration experiments. We conclude by noting that the soft elasticity of the SV solid should also manifest itself in many other physical observables, most notably in dynamics, exhibiting novel current-voltage characteristics and enhanced critical currents, sensitive to and with nontrivial dependence on $H$.

We thank Anton Andreev and Tom Lubensky for discussions and acknowledge financial support by the NSF through DMR99-80123 (J. T., K. S.), DMR96-25111, MRSEC DMR-9809555, and the A.P. Sloan and The David and Lucile Packard Foundations (L. R., M.E.).

[1] P. Canfield et al., Phys. Today 51, No. 10, 40 (1998).

[2] U. Yaron et al., Nature (London) 386, 236 (1996).

[3] H. S. Greenside, E. I. Blount, and C. M. Varma, Phys. Rev. Lett. 46, 49 (1981); T. K. Ng and C. M. Varma, ibid. 78, 330 (1997); 78, 3745 (1997).

[4] A. I. Larkin and Yu. N. Ovchinnikov, J. Low Temp. Phys. 34, 409 (1979).

[5] Such behavior contrasts strongly from the large $k$ ( $k \lambda_{L}>$ 1) wave-vector dependence of the elastic moduli due to the finite vortex interaction range $\lambda_{L}$ as discussed by E. H. Brandt, J. Low Temp. Phys. 26, 709 (1977); 26, 735 (1977); 28, 263 (1977); 28, 291 (1977).

[6] G. Blatter et al., Rev. Mod. Phys. 66, 1125 (1994).

[7] T. C. Lubensky et al., Phys. Rev. A 43, 5449 (1991).

[8] K. Saunders, L. Radzihovsky, J. Toner, and M. Ettouhami (unpublished).

[9] L. Radzihovsky and J. Toner, Phys. Rev. Lett. 79, 4214 (1997); 78, 4414 (1997); Phys. Rev. B 60, 206 (1999).

[10] D. S. Fisher, Phys. Rev. B 31, 7233 (1985); J. Villain and J. F. Fernandez, Z. Phys. B 54, 416 (1986).

[11] B. Jacobsen, K. Saunders, L. Radzihovsky, and J. Toner, Phys. Rev. Lett. 83, 1363 (1999).

[12] K. Saunders, L. Radzihovsky, and J. Toner, Phys. Rev. Lett. 85, 4309 (2000).

[13] T. Giamarchi and P. Le Doussal, Phys. Rev. Lett. 72, 1530 (1994).

[14] D. S. Fisher, Phys. Rev. Lett. 78, 1964 (1997); M. J. P. Gingras and D. A. Huse, Phys. Rev. B 53, 15193 (1996). 